\title{
Dynamic Relative Gain Array Estimation using Local Polynomial Approximation Approach
}

\author{
Ali M. H. Kadhim ${ }^{1}{ }^{2}$ Wolfgang Birk ${ }^{1}$ Miguel Castaño Arranz ${ }^{1}$ \\ ${ }^{1}$ Control Engneering Group, Signals and Systems Division. Luleå University of Technology, Luleå, Sweden. \\ E-mail: \{ali.kadhim,wolfgang.birk,miguel.castano\} @ltu.se \\ ${ }^{2}$ Electrical Engineering Department, Faculty of Engineering, University of Kufa, Najaf, Iraq.
}

\begin{abstract}
This article presents a procedure that utilizes the local polynomial approximation approach in the estimation of the Dynamic Relative Gain Array (DRGA) matrix and its uncertainty bounds for weakly nonlinear systems. This procedure offers enhanced frequency resolution and noise reduction when random excitation is used. It also allows separation of nonlinear distortions with shorter measuring time when multisine excitation is imposed. The procedure is illustrated using the well-known quadruple tank process as a case study in simulation and in real life. Besides, a comparison with the pairing results of the static RGA, nonlinear RGA and DRGA based on linearized quadruple tank model for different simulation cases is performed.
\end{abstract}

Keywords: Dynamic Relative Gain Array, nonparametric identification, local polynomial approximation approach, weakly nonlinear systems.

\section{Introduction}

Decentralized controllers are usually preferred in controlling industrial plants, as their design would eventually reduce to designing single-input single output (SISO) controllers. The individual controllers are also easier to maintain and update than the multivariable ones (Goodwin et al., 2001). It is also well-known that the choice of the inputs and outputs pairs affects the achievable performance of the decentralized control system, which makes the selection of inputoutput pairs a crucial design step. A systematic and reliable procedure for the selection of the pairs is therefore needed in order to achieve the desired performance goals for a decentralized control system. Therefore, efforts to develop pairing techniques have been carried on since the pioneer work of Bristol on the Relative Gain Array (RGA) in 1966 (Bristol, 1966). The RGA provides a method to select the input-output pairs for multi-loop SISO controllers by means of the steady- state gain matrix of the square linear systems.

Later on, many extensions were developed such as the Dynamic Relative Gain Array (DRGA) proposed by Witcher in Witcher and McAvoy (1977) where the transfer function rather than the steady-state gain matrix was used. A comprehensive study on the extensions and variants can be found in Khaki-Sedigh and Moaveni (2009). Since all real systems are nonlinear to some extent, RGA is still adapted in addressing the pairing selection for those systems. On the one hand, the original RGA formula, is applied on the linearized parametric model around a specified operating point. On the other hand, nonlinear RGA formula, applied directly to the nonlinear systems models, is derived in Glad (2000) and updated in Moaveni and Khaki-Sedigh (2007) resulting in a general approach to input-output pairing for linear and nonlinear systems following the relative gain definition. However, such approaches are limited to systems with accurately known models.

Aiming at taking the system dynamics into consid- 
eration in the input-output pairing decision, DRGA rather than RGA values are usually used. The common procedure to calculate DRGA values for systems with unknown model is to use a parametric model obtained from system identification techniques. Therefore, the user needs to decide on a model structure and model order, and subsequently to calculate the Frequency Response Matrix (FRM). Eventually, the DRGA values are obtained over a frequency range of interest. The wrong choice of the model structure and/or the model order has an influence on the quality of the system representation (Söderström and Stoica, 1988) and consequently on the DRGA calculated values. Besides, other sources such as changes in the operating point or parameters values introduce uncertainties to the RGA/DRGA values. To quantify these uncertainties, an analytical expression of the worst-case RGA as well as statistical RGA bounds for $2 \times 2$ and $n \times n$ uncertain systems are derived in Chen and Seborg (2002). A tighter bound of the worst-case RGA using the structured singular value is proposed in Kariwala et al. (2006). However, for large systems, this bound is computationally expensive and a simpler-calculated bound was proposed by Kadhim et al. (2015a). The results of Chen and Seborg (2002), Kariwala et al. (2006) and Kadhim et al. (2015a) can straightforwardly be extended to the DRGA.

In previous work, (Kadhim et al., 2014) and (Kadhim et al., 2015b), the authors have investigated an approach that seems obvious yet was not thoroughly discussed before which requires less user interaction and efforts in estimating RGA and DRGA. The approach employs a nonparametric system identification method to estimate the system Frequency Response Matrix (FRM) from input-output data and then calculates RGA and DRGA. Consequently, from the experimental data, the controller configuration can be directly decided. Such an approach reduces the uncertainties arising from incorrect user decisions by avoiding the parametric model identification. In Kadhim et al. (2014), both RGA and DRGA of linear systems are first determined using random excitation signal. Following the approach defined in Pintelon and Schoukens (2012), data is divided into sub-records and the frequency response is averaged over these sub-records to reduce the effect of the leakage that result from the nonperiodic nature of the random signal. Although the data division proved to be efficient in limiting the leakage effect, it has a drawback of reducing the frequency resolution of the result. However, a multisine rather than random excitation signal is used to determine DRGA of weakly nonlinear systems in Kadhim et al. (2015b). The uncertainties of the DRGA values are then obtained following the derivation in Chen and Seborg (2002). The multisine excitation simplifies the distinction between the nonlinear distortion and the output noise which is difficult to achieve using random excitation (Pintelon and Schoukens, 2012) as well as it improves the signal to noise ratio (SNR) (Schoukens et al., 2010). Unfortunately, this comes with the cost of requiring long experimental time for multi-input multi-output (MIMO) systems.

This work aims to overcome the shortcomings represented by the low frequency resolution in Kadhim et al. (2014) and the long experiment running time in Kadhim et al. (2015b) while preserving the advantages of using the nonparametric identification approach in DRGA calculation. To achieve this for weakly nonlinear systems, the Local Polynomial Approximation Method (LPA) and the Local Polynomial Approximation-Fast Method (LPA-FM) introduced in Pintelon and Schoukens (2012) are employed with both random and multisine excitation signals, respectively. This results in estimating the best linear approximation $\left(G_{B L A}\right)$ and its covarience caused by the output noise and the nonlinear distortion. Hence, DRGA values and their uncertainty bounds can be directly calculated using the estimated frequency response and the estimated covariance of the $G_{B L A}$. To make the decision more robust against the uncertainty sources, i.e the noise and the nonlinear distortions, the uncertainty bound of the DRGA are taken into considerations. The proposed procedure is applied on a case study of a quadruple tank process in simulation and on real plant to discuss the applicability of the different nonparametric identification techniques in the input-output pairing selection area.

The article is structured so that a brief definition of the RGA, DRGA, the pairing rules and the uncertainty in DRGA values are given in the following Preliminaries section. Theoretical background and the algorithm of applying the proposed procedure are given in sections 3 and 4, respectively. The advantages of implementing the proposed approach in simulation and on real plant are discussed from two prospectives in two separate sections. Enhancing the frequency resolution and reducing the noise effect are discussed in section 5 whereas section 6 deals with the separation of the nonlinear distortions. Finally, conclusions are drawn and future work directions are given in the last section.

\section{Preliminaries}

\subsection{Definition of RGA, DRGA and Pairing Rules}

The Relative Gain Array (RGA) elements $\left(\lambda_{i j}\right)$ are defined as the gain between input $u_{j}$ and output $y_{i}$ 
when all other loops are opened divided by the gain between the same input and output when all other loops are closed under perfect control assumption (Grosdidier et al., 1985). Provided that the steadystate gain matrix $G(0)$ of the systems is given, the RGA values can easily be obtained by applying

$$
\Lambda(0)=G(0) \times G(0)^{-T}
$$

where $\times$ denotes an element-by-element product and $-T$ is the inverse transpose of the matrix. Despite the fact that RGA is essentially proposed at steady-state (Bristol, 1966), (1) is usually used for any frequency in the range of interest (Witcher and McAvoy, 1977) and hence it can be referred to as Dynamic Relative Gain Array (DRGA).

For input-output pairing, there are rules to be followed (Khaki-Sedigh and Moaveni, 2009):

1. Choose the input-output pairs that correspond to the RGA elements close to 1.

2. Avoid pairing with negative or large RGA elements.

\subsection{Uncertainty in the DRGA results}

Since the system models are never perfect, research efforts are exerted to quantify the uncertainty effect on the RGA results. The most important result was proposed by Chen and Seborg in Chen and Seborg (2002) based on statistical approach. In that work, a closed form of the variance of RGA elements, denoted by $\sigma_{\lambda_{i j}}^{2}$, was derived. An extension of the result to frequency $\omega$ is stated in the following lemma

Lemma. For $2 \times 2$ system, where the nominal gains $\tilde{G}(\omega)$ and the covariance matrices $\operatorname{Cov}(G(\omega))$ at frequency $\omega$ are given and E denotes the expectation operator, the $\sigma_{\lambda_{i j}}^{2}(\omega)$ can be approximated as in (2)

where $\frac{\partial \lambda_{i j}(\omega)}{\partial G_{i j}(\omega)}$ is calculated as (Grosdidier et al., 1985)

$$
\frac{\partial \lambda_{i j}(\omega)}{\partial G_{i j}(\omega)}=\frac{\left(1-\lambda_{i j}(\omega)\right) \lambda_{i j}(\omega)}{G_{i j}(\omega)}
$$

\section{Theoretical Background}

The definition of the weakly nonlinear system as well as an overview of the identification methods (Spectral Analysis and Local Polynomial Approximation) are given in this section.

\subsection{Weakly nonlinear systems}

This study considers the class of nonlinear systems where the outputs can be well approximated by volterra series including hard nonlinear systems such as saturation, clipping and dead zone (Schoukens et al., 2014).

Definition. The weakly nonlinear system is defined as the nonlinear system where the coefficients of the first-order kernels of the volterra series dominate over the coefficients of the higher order kernels (Dobrowiecki and Schoukens, 2007).

Following the definition, a weakly nonlinear system can be described by a linear model since the linear contribution in the output is dominating the nonlinear distortions. The disturbed output of weakly nonlinear systems, excited by a class of Gaussian excitation (Gaussian noise, periodic Gaussian noise, random phase multisine), can be approximated in least square sense by an output of a linear model as (Pintelon et al., 2010)

$$
y(t)=g_{B L A} * u(t)+y_{s}(t)+v(t)
$$

where $*$ is the convolution product, $v(t)$ is the filtered output noise, $g_{B L A}$ is the impulse response of the best linear approximation $G_{B L A}$ and $y_{s}(t)$ represents the portion of the nonlinear output that is not captured by the linear model $G_{B L A}$, see Figures 1 and 2 . The

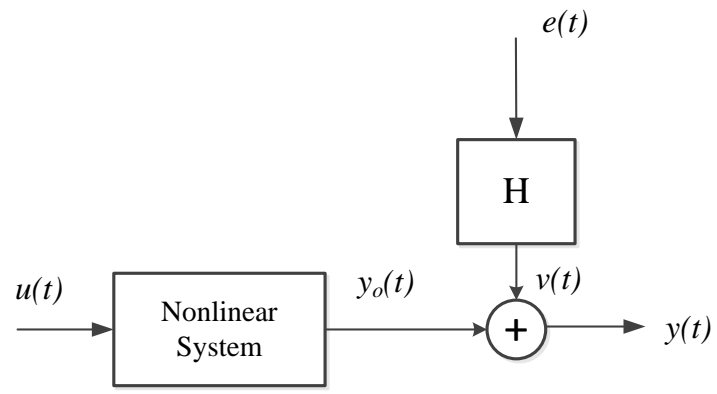

Figure 1: Nonlinear system.

$G_{B L A}$ is defined as a linear system whose output is as close as possible, in the mean square error sense, to the output of the nonlinear system (Schoukens et al., 2014).

$$
\sigma_{\lambda_{i j}}^{2}(\omega)=\mathrm{E}\left[\left(\lambda_{i j}(\omega)-\tilde{\lambda}_{i j}(\omega)\right)^{2}\right] \approx \sum_{k=1}^{2} \sum_{l=1}^{2} \sum_{m=1}^{2} \sum_{n=1}^{2}\left(\frac{\partial \lambda_{i j}(\omega)}{\partial G_{k l}(\omega)}\right)_{\tilde{G}}\left(\frac{\partial \lambda_{i j}(\omega)}{\partial G_{m n}(\omega)}\right)_{\tilde{G}} \operatorname{Cov}(G(\omega))
$$




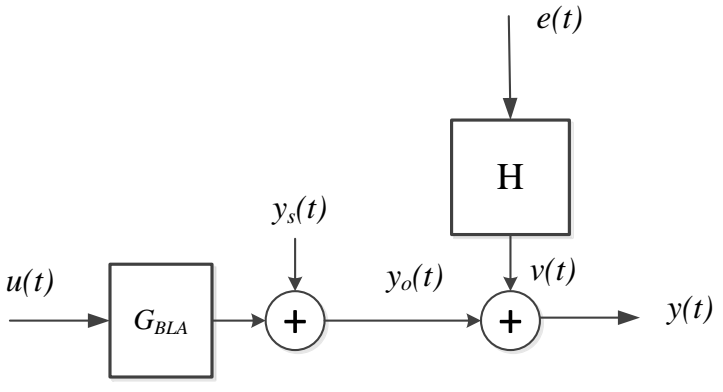

Figure 2: Best linear approximation of the weakly nonlinear system.

\subsection{Spectral Analysis Method (SA)}

For the system shown in Figure 2, the input-output Discrete Fourier Transform (DFT) spectra $U(k), Y(k)$ are related in DFT frequency $k$ as follows (Pintelon and Schoukens, 2012)

$$
\begin{aligned}
Y(k)=G_{B L A}\left(\omega_{k}\right) U(k) & +Y_{s}(k)+T_{G}\left(\omega_{k}\right) \\
& +H\left(\omega_{k}\right) E(k)+T_{H}\left(\omega_{k}\right)
\end{aligned}
$$

where $\omega_{k}=2 \pi k f_{s} / N$ and $f_{s}=1 / T_{s}$ with $T_{s}$ being the sampling time. $G_{B L A}\left(\omega_{k}\right)$ and $H\left(\omega_{k}\right)$ are the frequency response matrices of the best linear approximation of the system and the noise at DFT line $k$, respectively. The term $H\left(\omega_{k}\right) E(k)$ is usually abbreviated by $V(k)$. Moreover, for the same DFT line $k, T_{G}\left(\omega_{k}\right)$ and $T_{H}\left(\omega_{k}\right)$ represent the leakage error for system and noise respectively, while $Y_{s}(k)$ represents the nonlinear distortion in frequency response measurement.

For general nonlinear $n_{y} \times n_{u}$ system depicted in Figure $1, \hat{G}_{B L A}$ at DFT line $k$ can be estimated as (Pintelon and Schoukens, 2012)

$$
\hat{G}_{B L A}\left(\omega_{k}\right)=\hat{S}_{Y U}(k) \hat{S}_{U U}^{-1}(k)
$$

where $\hat{S}_{Y U}$ and $\hat{S}_{U U}$ are the estimated cross and autopower spectra, respectively. To reduce the leakage effect, the collected data is divided into $M$ blocks (subrecords) then averaged in the estimation of $\hat{S}_{Y U}$ and $\hat{S}_{U U}$ at line $k$ (Schoukens et al., 2012).

The covariance of the noise $V$ at line $k$ can be estimated as (Schoukens et al., 2012)

$$
\hat{C}_{V}(k)=\frac{M}{M-n_{u}}\left(\hat{S}_{Y Y}(k)-\hat{S}_{Y U}(k) \hat{S}_{U U}^{-1}(k) \hat{S}_{Y U}^{H}(k)\right)
$$

with $q=M-n_{u}$ the number of the degrees of freedom (dof) and $H$ denotes a Hermitian transpose of the matrix. Hence, the covariance of the $\hat{G}_{B L A}$ is obtained as

$$
\operatorname{Cov}\left(\operatorname{vec}\left(\hat{G}_{B L A}\left(\omega_{k}\right)\right)\right) \approx \frac{1}{M} \hat{S}_{U U}^{-1}(k) \otimes \hat{C}_{V}(k)
$$

where $\otimes$ is the Kronecker product and (vec) puts the columns of the matrix on top of each other.

As the random excitation is used, there is no easy way to distinguish the nonlinear distortion $y_{s}(t)$ at the output measurement from the noise $v(t)$ in Figure 2 (Schoukens et al., 2014). Thus, the estimated noise covariance $\left(\hat{C}_{V}\right)$ in $(7)$ accounts for both nonlinear and noise distortions. It is worth mentioning that reducing the leakage effect by dividing the data to $M$ blocks renders a lower frequency resolution and introduces a trade-off situation. To overcome this shortcoming, Local Polynomial Approximation method is to be utilized.

\subsection{Local Polynomial Approximation Method (LPA)}

The basic idea of LPA is using Taylor series expansion to approximate $G_{B L A}\left(\omega_{k \pm r}\right)$ and $T\left(\omega_{k \pm r}\right)$ in (5) for $r=0,1, . ., n$ by a low order polynomial at DFT frequency $k$ where $T\left(\omega_{k \pm r}\right)$ is the summation of $T_{G}\left(\omega_{k \pm r}\right)$ and $T_{H}\left(\omega_{k \pm r}\right)$. The coefficients of the polynomial are estimated from the DFT of the input-output data via linear least square fit. Such an approximation is valid since $G_{B L A}(\omega)$ and $T(\omega)$ are considered to be smooth transfer functions having continuous derivative up to any order (Pintelon and Schoukens, 2012). The result is the estimation of $\hat{G}_{B L A}\left(\omega_{k}\right)$ and $\hat{T}\left(\omega_{k}\right)$ at DFT line $k$, whilst the noise covariance at line $k$ can be estimated from the residual of the least square fit. The estimated noise covariance comprises the effect of both the nonlinear and the noise distortions since a random excitation signals is used here.

The estimation sequence is repeated for DFT frequency $k+1$ to estimate $\hat{G}_{B L A}\left(\omega_{k+1}\right), \hat{T}\left(\omega_{k+1}\right)$ and $\hat{C}_{V}(k+1)$ as well as all frequencies in the band of interest (Pintelon et al., 2010). The procedure is summarized from Pintelon and Schoukens (2012) as follows

Rewriting (5) after approximating $G_{B L A}\left(\omega_{k+r}\right)$ and $T\left(\omega_{k+r}\right)$ by polynomial of order $R$ at DFT frequency $k$, it can be expressed in the form

$$
\begin{aligned}
Y(k+r)=\left(G_{B L A}\left(\omega_{k}\right)\right. & \left.+\sum_{s=1}^{R} g_{s}(k) r^{s}\right) U(k+r)+T\left(\omega_{k}\right) \\
& +\sum_{s=1}^{R} t_{s}(k) r^{s}+V(k+r) \\
=\Theta K(k+r) & +V(k+r)
\end{aligned}
$$

where $\Theta$ is the $n_{y} \times(R+1)\left(n_{u}+1\right)$ matrix of unknown complex parameters

$$
\begin{aligned}
& \Theta=\left[\begin{array}{lllll}
G_{B L A}\left(\omega_{k}\right) & g_{1}(k) & g_{2}(k) & \cdots & g_{R}(k)
\end{array}\right. \\
& \left.T\left(\omega_{k}\right) \quad t_{1}(k) \quad t_{2} \quad \cdots \quad t_{R}(k)\right]
\end{aligned}
$$


and $K(k+r)$ is the $(R+1)\left(n_{u}+1\right) \times 1$ input data vector. Notice that, the contribution of $Y_{s}(k+r)$ has been included in the $V(k+r)$ since the random excitation signals are used. Redoing (9) for $r=-n,-n+$ $1, \cdots, 0, \cdots, n-1, n$ results in

$$
Y_{n}=\Theta K_{n}+V_{n}
$$

where $Y_{n}, K_{n}$ and $V_{n}$ sizes are $n_{y} \times(2 n+1),(R+$ 1) $\left(n_{u}+1\right) \times(2 n+1)$ and $n_{y} \times(2 n+1)$, respectively. If $2 n+1 \geq(R+1)\left(n_{u}+1\right), \Theta$ can be estimated from (11) using least square method as

$$
\hat{\Theta}=Y_{n} K_{n}^{H}\left(K_{n} K_{n}^{H}\right)^{-1}
$$

The noise covariance can be estimated form the residual of the fitting as

$$
\begin{aligned}
\hat{V}_{n} & =Y_{n}-\hat{\Theta} K_{n} \\
\hat{C}_{V}(k) & =\frac{1}{q} \hat{V}_{n} \hat{V}_{n}^{H}
\end{aligned}
$$

where $q=\left(2 n+1-(R+1)\left(n_{u}+1\right)\right)$ is the number of dof of $\hat{V}_{n}^{H}$.

Then, $\hat{G}_{B L A}$ and its covariance matrix at DFT frequency $k$ can be estimated as (Pintelon and Schoukens, 2012)

$$
\hat{G}_{B L A}\left(\omega_{k}\right)=\hat{\Theta}\left[\begin{array}{c}
I_{n_{u}} \\
0
\end{array}\right]
$$

which selects the first $n_{u}$ columns of $\hat{\Theta}$ matrix, and

$$
\operatorname{Cov}\left(\operatorname{vec}\left(\hat{G}_{B L A}\left(\omega_{k}\right)\right)\right) \approx \overline{S^{H} S} \otimes \hat{C}_{V}(k)
$$

where $S=K_{n}^{H}\left(K_{n} K_{n}^{H}\right)^{-1}\left[\begin{array}{c}I_{n_{u}} \\ 0\end{array}\right]$.

\section{Algorithm of the Proposed Procedure}

The procedure of estimating DRGA and its uncertainty bounds for weakly nonlinear systems can be summarized as in Algorithm 1. Notice that, in order to track the occurrences of the sign change in DRGA values, the pairing decision is to be made based on the real part of the estimated DRGA in frequency $\omega$. Notice that this algorithm does not apply for the separation of nonlinear distortions from the output noise which will be discussed later.

\section{Enhancing Frequency Resolution and Reducing Noise Effect}

In this section, LPA method is applied both in simulation and real life quadruple tank plant subject to random excitation. For comparison purposes SA method is applied in the simulation study.

\subsection{Simulation Study}

The quadruple tank process working around a specified operating point is employed to illustrate the DRGA calculation procedure since it behaves as a weakly nonlinear system when subject to Gaussian excitation (Arranz and Birk, 2015). To give a comprehensive understanding, the results of the estimated DRGA $(\hat{\Lambda})$ are compared to the results obtained by the nonlinear RGA $\left(\Lambda_{n l-R G A}\right)$ and with both RGA $(\Lambda(0))$ and DRGA $(\Lambda)$ based on a linearized transfer function $\left(G_{l i n}\right)$ of the physical model around a selected operating point.

The quadruple tank physical model based on mass balance and Bernoulli's principle is given by (Johansson, 2000)

$$
\begin{aligned}
& \dot{h_{1}}=-\frac{a_{1}}{A_{1}} \sqrt{2 g h_{1}}+\frac{\gamma_{1} k_{1}}{A_{1}} u_{1} \\
& \dot{h_{2}}=-\frac{a_{2}}{A_{2}} \sqrt{2 g h_{2}}+\frac{\gamma_{2} k_{2}}{A_{2}} u_{2} \\
& \dot{h_{3}}=-\frac{a_{3}}{A_{3}} \sqrt{2 g h_{3}}+\frac{a_{1}}{A_{3}} \sqrt{2 g h_{1}}+\frac{\left(1-\gamma_{2}\right) k_{2}}{A_{3}} u_{2} \\
& \dot{h_{4}}=-\frac{a_{4}}{A_{4}} \sqrt{2 g h_{4}}+\frac{a_{2}}{A_{4}} \sqrt{2 g h_{2}}+\frac{\left(1-\gamma_{1}\right) k_{1}}{A_{4}} u_{1}
\end{aligned}
$$

where the water heights of the $3^{\text {rd }}$ and $4^{\text {th }}$ tanks $\left(h_{3}\right.$ and $\left.h_{4}\right)$ are considered as the system outputs. $\gamma_{1}$ and $\gamma_{2}$ are valves openings splitting the water between tanks 1 and 4 and tanks 2 and 3, respectively as shown schematically in Figure 3. $u_{1}$ and $u_{2}$ are the input voltages driving the pumps 1 and 2 respectively. The description and the values of the parameters ${ }^{1}$ used in the simulation are tabulated in Table 2.

By manipulating the splitting valves openings, different scenarios can be achieved such as minimum phase, non-minimum phase, ill-conditioned, lower and upper triangular plants. In the following subsections, estimation of the DRGA for the mentioned scenarios is presented with a special focus on the minimum-phase case.

Table 1: Water levels in the quadruple tank for the selected operating point.

\begin{tabular}{cccc}
\hline$h_{1}(\mathrm{~cm})$ & $h_{2}(\mathrm{~cm})$ & $h_{3}(\mathrm{~cm})$ & $h_{4}(\mathrm{~cm})$ \\
\hline 7.5 & 6.5 & 7 & 6.5 \\
\hline
\end{tabular}

\subsubsection{Minimum-phase case}

To achieve the minimum-phase case, the splitting valves $\gamma_{1}$ and $\gamma_{2}$ are chosen to be $80 \%$ opened (Johansson, 2000). The presented operating point for this case in Table 1 renders a linearized transfer function $\left(G_{l i n}\right)$ as

$$
G_{\text {lin }}=\left[\begin{array}{cc}
\frac{2.516}{5000 s^{2}+142.27 s+1} & \frac{0.473}{63.45 s+1} \\
\frac{0.585}{75 s+1} & \frac{1.761}{7347.5 s^{2}+172.81 s+1}
\end{array}\right]
$$

\footnotetext{
${ }^{1}$ The parameters values are estimated based on the real quadruple tank process depicted in Figure 6.
} 


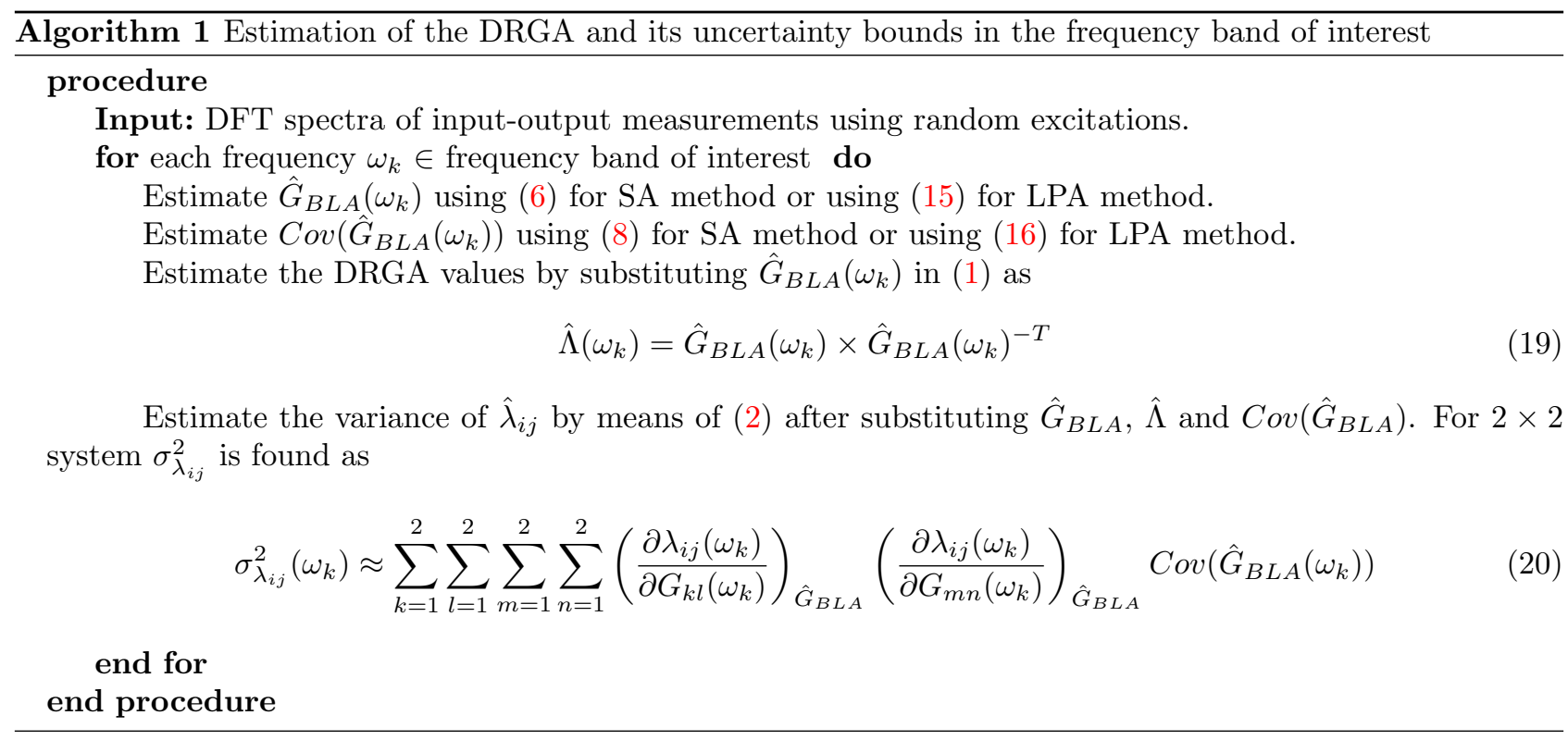

Table 2: Parameters values and description for quadruple tank process.

\begin{tabular}{ccc}
\hline Parameter & Values & Description \\
\hline$A_{1, . .4}$ & $28 \mathrm{~cm}^{2}$ & Cross section area of tank 1, 2, 3 and 4 \\
$a_{1}$ & $0.074 \mathrm{~cm}^{2}$ & Area of the hole of tank 1 \\
$a_{2}$ & $0.058 \mathrm{~cm}^{2}$ & Area of the hole of tank 2 \\
$a_{3}$ & $0.089 \mathrm{~cm}^{2}$ & Area of the hole of tank 3 \\
$a_{4}$ & $0.075 \mathrm{~cm}^{2}$ & Area of the hole of tank 4 \\
$g$ & $981 \mathrm{~cm} / \mathrm{s}^{2}$ & Gravity acceleration \\
$k_{1}$ & $0.31 \mathrm{~cm}^{3} / \mathrm{Vs}$ & Flow to volt unit of pump 1 \\
$k_{2}$ & $0.23 \mathrm{~cm}^{3} / \mathrm{Vs}$ & Flow to volt unit of pump 2 \\
$k_{c 3}$ & $3.84 \mathrm{~V} / \mathrm{cm}$ & Constant of the level sensor of tank 3 \\
$k_{c 4}$ & $3.48 \mathrm{~V} / \mathrm{cm}$ & Constant of the level sensor of tank 4 \\
\hline
\end{tabular}


In this case, the diagonal pairing is suggested to con-

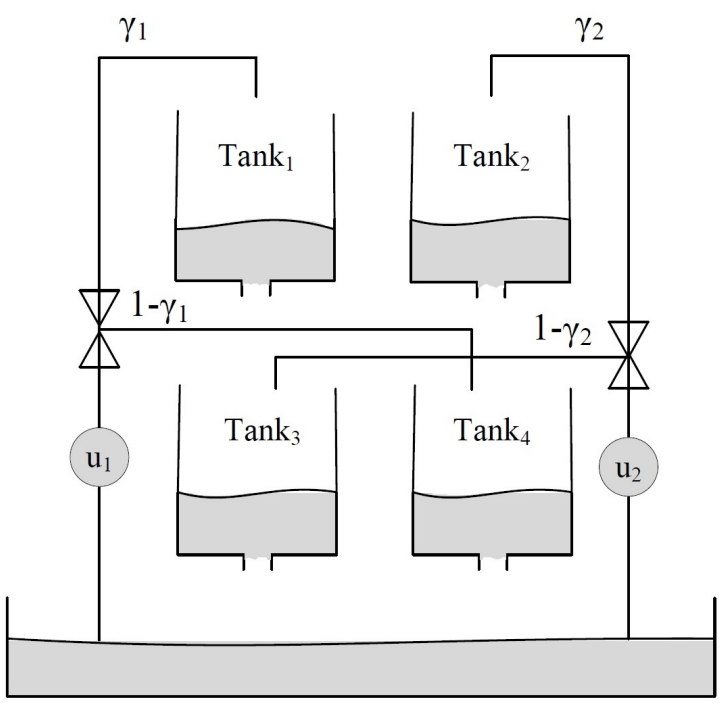

Figure 3: A sketch of the quadruple tank process.

trol the water levels $h_{3}$ and $h_{4}$ (Johansson, 2000). This pairing selection is quite intuitive since pumps 1 and 2 pump more water into tanks 1 and 2 respectively, thus controlling levels $h_{3}$ and $h_{4}$ through tanks 1 and 2 is preferable. The diagonal pairing suggestion is confirmed by $\Lambda(0)$ values obtained using an expression that relates $\left(\lambda_{11}(0)\right)$ element in the $\Lambda(0)$ matrix to the valves positions by (Johansson, 2000)

$$
\lambda_{11}(0)=\frac{\gamma_{1} \gamma_{2}}{\gamma_{1}+\gamma_{2}-1}
$$

Thus, $\lambda_{11}(0)$ will be equal to 1.066 which promotes the diagonal pairing according to the RGA pairing rules. Based on the pairing rules of $\Lambda_{n l-R G A}$ (Moaveni and Khaki-Sedigh, 2007), the diagonal pairing is also suggested since $\lambda_{11_{n l-R G A}}=1.118$.

\section{Excitation Signals}

Gaussian random excitation signals $\mathcal{N}(0,10)$ are generated with $N=5000$ samples and a sampling frequency $f_{s}=1 \mathrm{~Hz}$. After the simulated tank levels reach the operating point, the excitation signals are superimposed on $u_{1}$ and $u_{2}$. The outputs of the simulation, $h_{3}$ and $h_{4}$, are disturbed by filtered random Gaussian noise $\mathcal{N}(0,0.2)$ to simulate the measurement noise. Both the excitation signals and the responded noisy outputs are used to estimate the DRGA $(\hat{\Lambda})$ and its uncertainty bounds by means of SA and LPA methods.

\section{Spectral Analysis Method}

In order to reduce the leakage in the estimated results, the collected data are divided into $M$ blocks followed by averaging them to a single estimate. Selecting a suitable $M$ is a trade-off between the leakage elimination and the frequency resolution from one side and the noise suppression from the other (Pintelon and Schoukens, 2012). Therefore, $M$ should be selected as small as possible as well as it should satisfy the condition $q=M-n_{u} \geq n_{y}$ (Pintelon and Schoukens, 2012). Thus, $M$ is selected to be 4 since both $n_{u}$ and $n_{y}$ are equal to 2 . Therefore, the frequency resolution decreased from $f_{s} / N$ to $M f_{s} / N$. Moreover, to suppress the leakage effect on the DFT spectra, windows other than rectangular window are usually applied to the time domain, such as Hanning, diff or half-sine windows. In Pintelon et al. (2010), the system and the noise leakage error of diff and half-sine windows are shown to be greater than Hanning window, while the interpolation error of Hanning is greater than that in diff and half-sine windows. The results of Antoni and Schoukens (2007) show that diff window is optimal in the estimation of the frequency response function which motivates its usage in the current simulations. Following Algorithm 1, the FRM of $G_{B L A}$ and its covariance are estimated (see Figure $4 \mathrm{a}$ ) and then used in the estimation of the real parts of $\hat{\lambda}_{11}$ and the $\pm 3 \sigma_{\lambda_{11}}$ uncertainty bounds (see Figure $4 \mathrm{~b}$ ). The real parts of $\lambda_{11}$ calculated from the linearized parametric model (18) are also illustrated in Figure 4b. The pairing suggestion of $\hat{\lambda}_{11}$ coincides with $\lambda_{11}(0)$ and $\lambda_{11 n l-R G A}$ for the low frequencies with recommendation of the diagonal pairing while it shows completely different suggestion in frequencies higher than $0.008 \mathrm{~Hz}$ promoting an off-diagonal pairing. That behaviour is physically explainable since opening the splitting valves by $80 \%$ $\left(\gamma_{1}=\gamma_{2}=0.8\right)$ means more water flow goes to the upper tanks which allows easier controlling of water levels in the lower tanks using diagonal pairing. Even when involving the upper tanks dynamics such pairing gives an acceptable performance with slow references changes such as step changes. On the other hand, when the references frequency increases, it is easier for the levels of the lower tanks to keep tracking of the references through the direct water pumping even with the small splitting valve opening $\left(1-\gamma_{1}=1-\gamma_{2}=0.2\right)$. In other words, avoiding the upper tanks dynamics with frequency increasing motivates off-diagonal pairing. This pairing suggestion is also confirmed by $\lambda_{11}$ obtained by the linearized parametric model, see Figure $4 \mathrm{~b}$. Moreover, although the value of $\hat{\lambda}_{11},(0.6)$ promotes diagonal pairing around $0.006 \mathrm{~Hz}$, the lower bound of the uncertainty bounds, [0.44 0.76$]$, reveals that a highly interaction effect is expected. Therefore, neither diagonal nor off-diagonal pairing can be suggested for that frequency range and a sparse or centralized controllers would be preferred. 

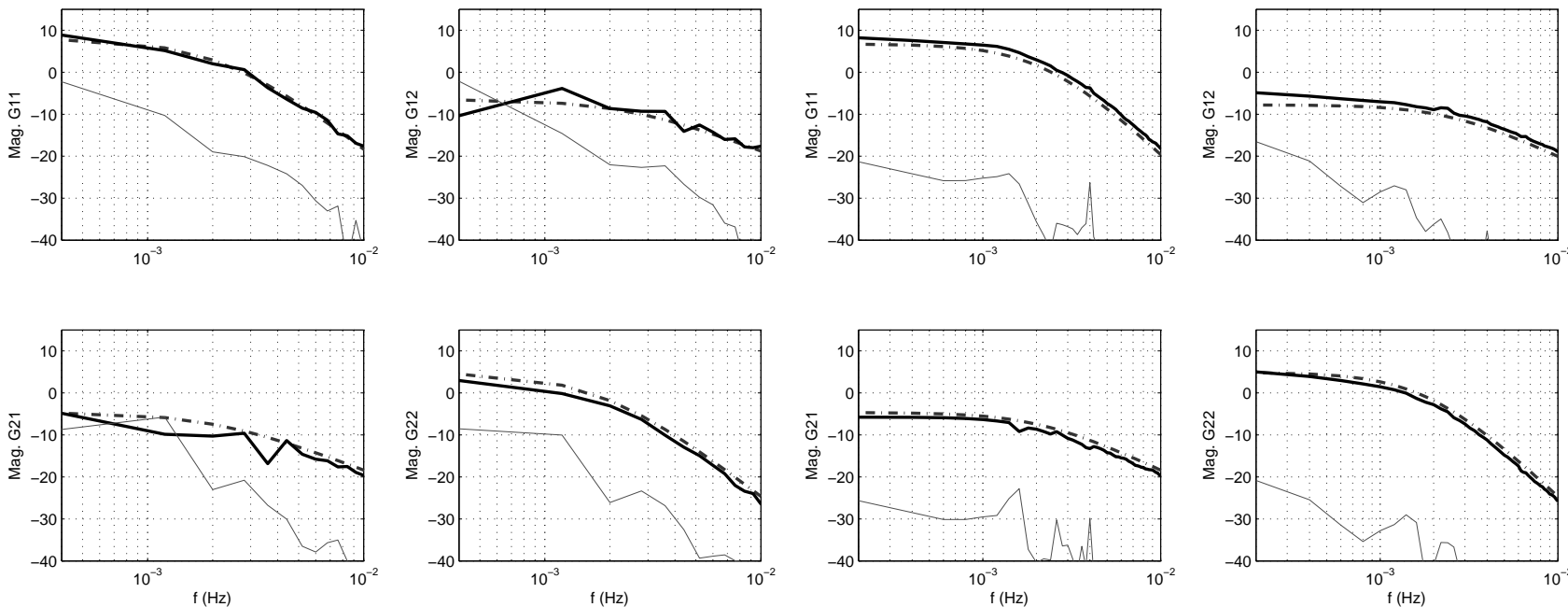

(a) Estimated FRM of $G_{B L A}$ using SA of the simulated case with random excitation.

Black: FRM of $\hat{G}_{B L A}$. light Gray: Variances of $\hat{G}_{B L A}$. dark Gray (-•): FRM of $G_{l i n}$.

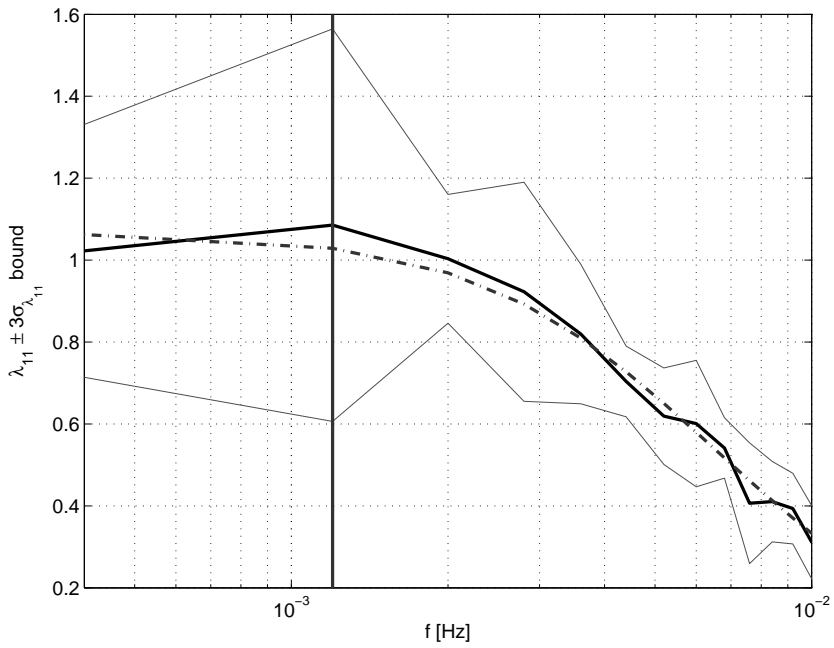

(b) Real parts of $\hat{\lambda}_{11}$ and $\pm 3 \sigma_{\lambda_{11}}$ bounds using SA of the simulated case with random excitation. Black: $\hat{\lambda}_{11}$. light Gray: $\hat{\lambda}_{11} \pm 3 \sigma_{\lambda_{11}}$ uncertainty bounds. dark Gray (-): $\lambda_{11}$ calculated based on $G_{l i n}$. Bold vertical line: indicates $0.0012 \mathrm{~Hz}$.
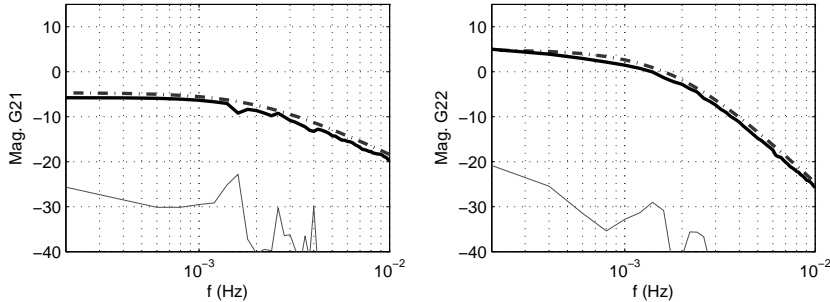

(a) Estimated FRM of $G_{B L A}$ using LPA of the simulated case with random excitation.

Black: FRM of $\hat{G}_{B L A}$. light Gray: Variances of $\hat{G}_{B L A}$. dark Gray (-.): FRM of $G_{l i n}$.

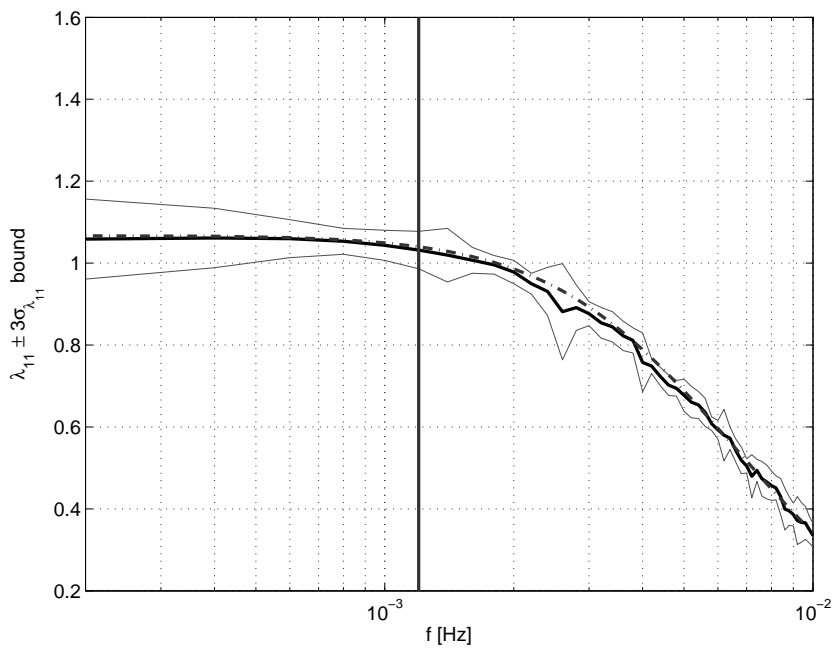

(b) Real parts of $\hat{\lambda}_{11}$ and $\pm 3 \sigma_{\lambda_{11}}$ bounds using LPA of the simulated case with random excitation. Black: $\hat{\lambda}_{11}$. light Gray: $\hat{\lambda}_{11} \pm 3 \sigma_{\lambda_{11}}$ uncertainty bounds. dark Gray (--): $\lambda_{11}$ calculated based on $G_{l i n}$. Bold vertical line: indicates $0.0012 \mathrm{~Hz}$.

Figure 4: Simulation results of $\hat{G}_{B L A}$ and $\hat{\lambda}_{11}$ using SA method.

Figure 5: Simulation results of $\hat{G}_{B L A}$ and $\hat{\lambda}_{11}$ using LPA method. 


\section{Local Polynomial Approximation Method}

To maintain the frequency resolution as $f_{s} / N$ and reduce the interpolation and leakage errors on both the estimated FRM of $G_{B L A}$ and its covariance, LPA method is used. In order to make a fair comparison with SA results, the order of the polynomial $(R)$ is selected to be 2 (lowest order possible) and the dof $(q)$ is selected to be 2 (equal to that in the SA case). The LPA method is applied to the same collected data used in the simulation of the SA method.

Figure 5a clearly shows the enhanced results of LPA method over SA method. The leakage and the noise reduction of this method can be noticed from the results of the variances of $\hat{G}_{B L A}$ shown in Figure 5 a compared to Figure 4a. More reduction in those variances can be achieved using LPA by increasing the degree of freedom, while increasing the degree of freedom in the SA results is reducing the frequency resolution in returns. Beside the higher resolution of the $\hat{\lambda}_{11}$, the uncertainty bounds are reduced significantly.

Despite the fact that the same pairing decisions are obtained based on $\hat{\lambda}_{11}$ values of both LPA and SA methods for low and high frequencies, the user can take more confident pairing decision based on LPA results since the uncertainty bounds are significantly reduced, see Figure 5b. For example, utilizing a diagonal decentralized controller for a closed-loop bandwidth around $0.0012 \mathrm{~Hz}$, the system would be mistaken to suffer performance degradation due to the uncertainty bounds of $\hat{\lambda}_{11},[0.621 .55]$, indicating largely interactive system in the SA results. Whereas the system is almost decoupled at that frequency based on the uncertainty bounds of $\hat{\lambda}_{11}$, [ 11 1.06], in the LPA method.

\subsubsection{Other Simulation Cases}

Non-minimum phase, lower triangular, upper triangular and ill-conditioned plant (with condition number value of 26) cases are also simulated in order to verify the results of the proposed procedure. These cases are easily achieved by changing the opening of the splitting valves $\gamma_{1}$ and $\gamma_{2}$. Combinations of splitting valves opening, the values of RGA $\left(\lambda_{11}(0)\right), \lambda_{11 n l-R G A}$ and $\hat{\lambda}_{11}$ with the standard deviation $\left(\sigma_{\lambda_{11}}\right)$ are tabulated in Table 3. Values of $\hat{\lambda}_{11}$ and $\sigma_{\lambda_{11}}$ are obtained using LPA method with both $R$ and $d o f$ equal to 2 for frequency $(f) 0.0002 \mathrm{~Hz}, 0.0014 \mathrm{~Hz}$ and $0.007 \mathrm{~Hz}$.

\section{Non-minimum case}

The negative or close to zero values of $\lambda_{11}$ for different frequencies of the proposed procedure coincide with the other methods and the intuition of having off-diagonal pairing. $80 \%$ of the amount of the water is pumped directly to the lower tanks from the opposite sources, i.e. most the water of the tank 3 and 4 come from pump 2 and 1, respectively (see Figure 3).

\section{Triangular cases}

The diagonal pairing is inevitable for the triangular cases (Khaki-Sedigh and Moaveni, 2009), the $\lambda_{11}$ values for the different methods are equal or close to 1 . From Figure 3, in the lower triangular case, tank 3 receives the water only from pump 1 hence $h_{3}$ can only be manipulated through that pump. Similarly, tank 4 in the upper triangular case receives the water from only pump 2 suggesting the diagonal pairing for all frequencies which confirms the results of $\hat{\lambda}_{11}$.

\section{III-conditioned case}

The pairing suggestions of the ill-conditioned case are quite different for the different pairing methods. On the one hand, RGA suggests diagonal pairing with an indication of difficult controlling since $\lambda_{11}(0)$ value is much higher than 1 . On the other hand, nonlinear RGA suggests off-diagonal pairing based on $\lambda_{11 n l-R G A}$ value $(-2.271)$. Both pairing suggestions appear in the result of the proposed method which favours the diagonal pairing for the frequencies close to the steady-state and off-diagonal for the middle and higher frequencies. The selection is similar to that of the minimum case yet it suggests the off-diagonal pairing in lower frequency $(0.0014 \mathrm{~Hz})$ since the opening of the direct splitting valves toward the lower tanks $\left(1-\gamma_{1}=1-\gamma_{2}=0.48\right)$ are bigger than that in the minimum phase case $(0.2)$. Moreover, the difference in the suggestions of the nonlinear RGA and RGA is a result of considering or neglecting the system dynamics, respectively. Finally, the high standard deviation in the estimated DRGA results is expected since the plant in the ill-conditioned case is sensitive to the noise and nonlinear distortion uncertainties.

\subsection{Real Life Study}

In order to apply the proposed procedure on real plant, a real quadruple tank process shown in Figure 6 in the minimum-phase case is employed. $u_{1}$ and $u_{2}$ were applied to drive the pumps to operate at $60 \%$ of their power until the equilibrium operating point was reached. With $80 \%$ opening of $\gamma_{1}$ and $\gamma_{2}$, the water levels in the tanks at that operating point are depicted in Table 1. Thereafter, the same random excitation inputs used in the simulation cases were applied. The random excitations are designed to be changed each $5 T_{s}\left(T_{s}=1 / f_{s}\right)$ to allow the quadruple tank to respond to that change. 
Table 3: Pairing suggestions of different plant cases based on different methods

\begin{tabular}{cccccccc}
\hline Plant Case & \multicolumn{2}{c}{ Opening } & \multicolumn{5}{c}{$\lambda_{11}$ values for different methods } \\
\hline & $\gamma_{1}$ & $\gamma_{2}$ & $\lambda_{11}(0)$ & $\lambda_{11 n l-R G A}$ & \multicolumn{3}{c}{$\left(\hat{\lambda}_{11}, \sigma_{\lambda_{11}}\right)$} \\
\cline { 6 - 8 } & & & & & $f=0.0002 H z$ & $f=0.0014 H z$ & $f=0.007 H z$ \\
\hline Non-minimum & 0.2 & 0.2 & -0.066 & -0.038 & $(-0.09,0.02)$ & $(-0.04,0.002)$ & $(0.004,0.001)$ \\
Lower triangular & 0.75 & 1 & 1 & 1 & $(1,0.01)$ & $(0.99,0.05)$ & $(1.02,0.03)$ \\
Upper triangular & 1 & 0.75 & 1 & 1 & $(0.99,0.07)$ & $(1,0.02)$ & $(1.03,0.2)$ \\
Ill-conditioned & 0.52 & 0.52 & 6.76 & -2.271 & $(3.4,0.9)$ & $(0.41,0.07)$ & $(0.108,0.009)$ \\
\hline
\end{tabular}

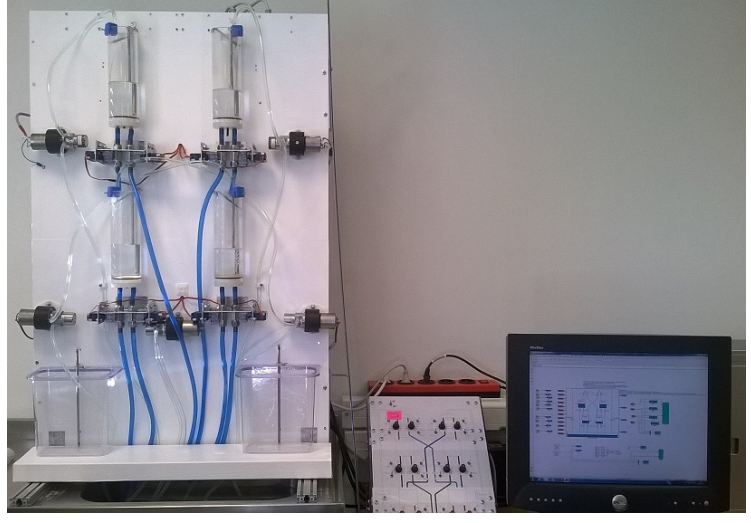

Figure 6: The quadruple tank process in the Control Engineering Group lab.

The LPA method is utilized in the estimation of DRGA with the values of polynomial order $(R)$ and $\operatorname{dof}(q)$ are selected to be 3 and 18, respectively. The real parts of $\hat{\lambda}_{11}, \hat{\lambda}_{11} \pm 3 \sigma_{\lambda_{11}}$ uncertainty bounds and the real part of $\lambda_{11}$ obtained from the linearized model (18) are illustrated in Figure 9a. The figure shows that the real parts of $\hat{\lambda}_{11}$ and $\lambda_{11}$ suggest the same pairing decision (diagonal pairing) up to a frequency $0.006 \mathrm{~Hz}$. For higher frequencies, discrepancy occurs between the results of the real plant and its model due to the negligence of the dynamics of some system parts such as pumps and sensors. As discussed for the model, a good performance is still expected when using a diagonal decentralized controller around a closed-loop bandwidth of $0.0012 \mathrm{~Hz}$ since the uncertainty bounds of $\hat{\lambda}_{11},[0.995$ 1.24], show a plant with low interaction.

\section{Separation of Nonlinear Distortions}

Separation of nonlinear distortions $y_{s}(t)$ and noise $v(t)$ is possible when a nonlinear system such as that shown in Figure 1 is excited by the multisine signal

$$
u(t)=\frac{1}{\sqrt{N}} \sum_{k=\frac{-N}{2}+1}^{\frac{N}{2}-1} U_{k} e^{j\left(2 \pi k \frac{t}{N}+\phi_{k}\right)}
$$

with amplitude $U_{-k}=U_{k}$ and randomly chosen phases $\phi_{-k}=\phi_{k}$ such that $E\left\{e^{j \phi_{k}}=0\right\}$. It follows that the DFT spectra of the input-output relation are as (Pintelon and Schoukens, 2012)

$$
Y(k)=G_{B L A}\left(\omega_{k}\right) U(k)+Y_{s}(k)+V(k)+T_{H}\left(\omega_{k}\right)
$$

The noise covariance $\left(C_{V}\right)$ can be estimated over $P$ periods of the same $u(t)$ realization knowing that $y_{s}(t)$ is uncorrelated with, yet dependent on $u(t)$ and does not change over these periods. Besides, measuring the system outputs for $M$ different $u(t)$ realizations allows estimating the covariance of the nonlinear distortion $\left(C_{Y_{s}}\right)$ since $y_{s}(t)$ changes from one realization to the other (Pintelon and Schoukens, 2012).

Distinction between $y_{s}(t)$ and the noise $v(t)$ for multivariable $n_{y} \times n_{u}$ system requires at least $M \times n_{u}$ experiments with $P$ periods after the transient. Hence, in order to quantify $Y_{s}$, the realization of excitation signal needs to be changed not only from input to input but also between the sub-experiments. For that purpose, excitation signal known as full orthogonal random phase multisine is used that is represented by

$\mathbf{U}^{[m, p]}=\left[\begin{array}{ccc}U_{1 k} e^{j\left(\phi_{1 k}+\alpha_{1 k}\right)} & \cdots & U_{1 k} e^{j\left(\phi_{1 k}+\alpha_{n_{u} k}\right)} \\ \vdots & \ddots & \vdots \\ U_{n_{u} k} e^{j\left(\phi_{n_{u} k}+\alpha_{1 k}\right)} & \cdots & U_{n_{u} k} e^{j\left(\phi_{n_{u} k}+\alpha_{n_{u} k}\right)}\end{array}\right]$

for $m=1, \ldots, M$ where $M \geq 2$ and $p=1, \ldots, P$ where $P \geq 2$ with $\alpha$ being uniformly distributed over $[0,2 \pi)$ (Schoukens et al., 2010)(Wernholt and Gunnarsson, 2006). However, this signal prolongs the measuring time and it is more convenient to use an alternative approach as discussed in the following section. 


\section{Local Polynomial Approximation-Fast Method (LPA-FM)}

This method has an advantage of using only one experiment with periods $P \geq 2$, thus only one column of (24) is needed rather than the $M \times n_{u}$ experiments. Two estimation phases are employed to estimate the frequency response of $G_{B L A}$, the noise and the nonlinear distortion covariance matrices. The first phase is used to nonparametrically suppress the noise leakage error $T_{H}$; while the second phase is employed to estimate the frequency response of the $G_{B L A}$, the sample total noise and the nonlinear distortion covariances. The two phases basically follow the same principle of the LPA method. For curtailment purposes, the procedure of this method is skipped here and can be found in Chapter 7, Section 7.3 in Pintelon et al. (2010).

\subsection{Simulation and Real life studies}

Multisine excitations are used in order to sort out the uncertainty in the DRGA values caused by the nonlinear distortions and output noise. Multisine excitations with $R M S=10$ are designed to excite the odd DFT lines $\frac{f_{s}}{N}, \frac{3 f_{s}}{N}, \frac{5 f_{s}}{N}, \frac{7 f_{s}}{N} \ldots . ., \frac{2499 f_{s}}{N}$ with $N=5000$ and $f_{s}=1 H z$. Two periods of these excitations $(P=2)$ are superimposed on $u_{1}$ and $u_{2}$ after the tank levels in simulation and real for the minimum-phase case reach the operating points in Table 1. In the simulation, the outputs $h_{3}$ and $h_{4}$ are disturbed by filtered random Gaussian noise $\mathcal{N}(0,0.5)$ to simulate the measurement noise. Both the excitation signals and the noisy outputs are used to estimate the DRGA and its uncertainty bounds by means of LPA-FM. The multisine excitation signals constitute one column of the excitation signal given in (24) which reduces the experiment time significantly compared to the methods used in Kadhim et al. (2015b). Applying LPA-FM on the simulated data, the FRM of the $G_{B L A}$ in addition to both the sample noise covariance $\left(C_{V}\right)$ and the covariance of the nonlinear distortions $\left(C_{Y_{s}}\right)$ are estimated, see Figure 7. The figure shows that the linear contribution is dominating the distortion caused by the nonlinearity at this operating points; therefore, it is sufficient to consider the linear model in the pairing decision for this case. Thereafter, DRGA and its bounds of uncertainties caused by noise and nonlinear distortions shown in Figure 8 are estimated by means of (19) and (20), by exploiting $C_{Y_{s}}$ and $C_{V}$ respectively in the Algorithm 1. The dof is selected to be 4 in this method which satisfies the condition $d o f \geq n_{u}+n_{y}$. The pairing suggestion coincides with the minimum-phase case using LPA method as it suggests the diagonal pairing at the low frequencies and off-diagonal pairing at the high frequencies.
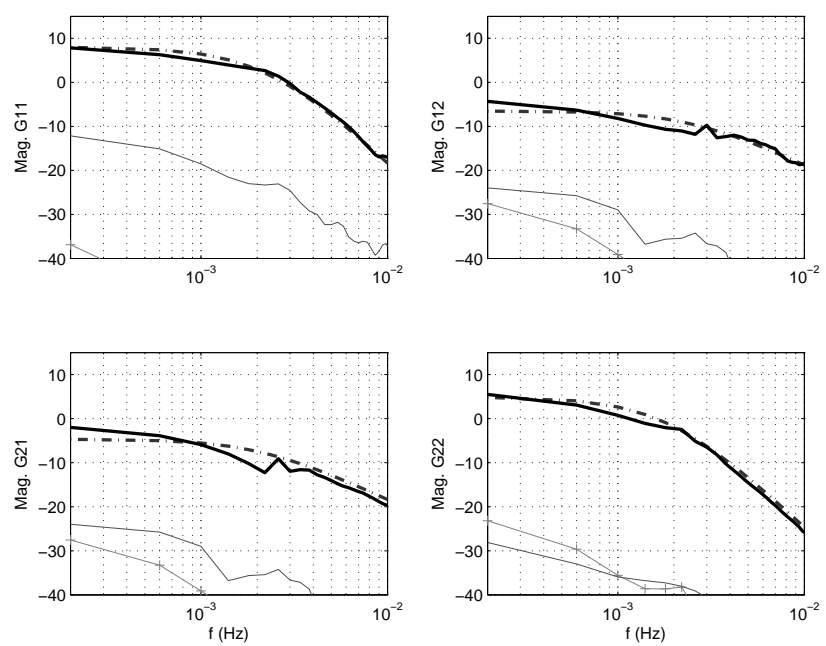

Figure 7: Estimated FRM of $G_{B L A}$ using LPA-FM of the simulated case with multisine excitation. Black: FRM of $\hat{G}_{B L A}$. Gray: Variances caused by noise. light Gray (-+): Variances caused by nonlinear distortions. dark Gray (-) : FRM of $G_{l i n}$.

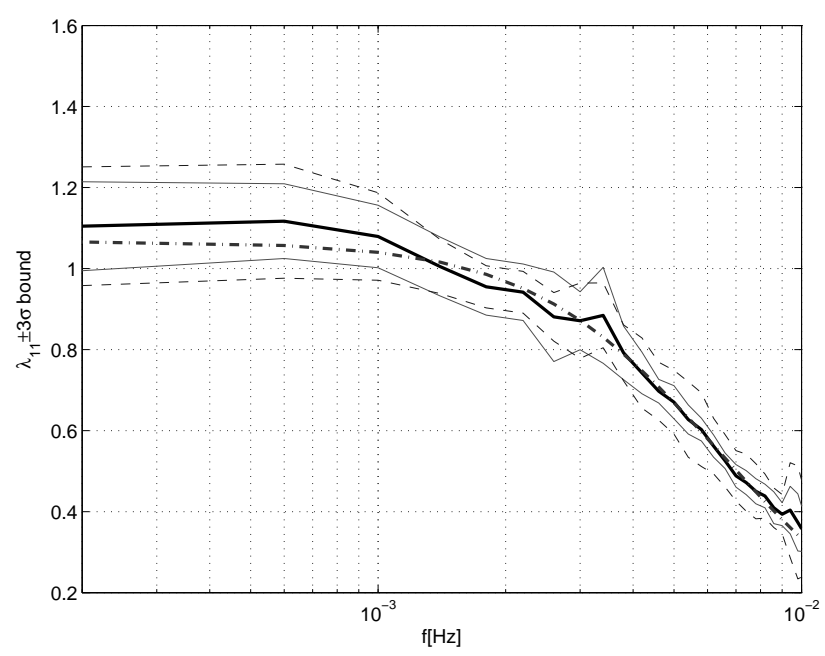

Figure 8: Real parts of $\hat{\lambda}_{11}$ and $\pm 3 \sigma_{\lambda_{11}}$ bounds using LPM-FM of the simulated case with multisine excitation. Black: $\hat{\lambda}_{11}$. Gray (- -): $\hat{\lambda}_{11} \pm 3 \sigma_{\lambda_{11}}$ uncertainty bounds caused by noise. light Gray: $\hat{\lambda}_{11} \pm 3 \sigma_{\lambda_{11}}$ uncertainty bounds caused by nonlinear distortions. dark Gray (-.): $\lambda_{11}$ calculated based on $G_{l i n}$. 
Although LPA-FM gives the user the privilege of distinguishing between the noise and the nonlinear contributions in the estimation, it needs twice as long experiment times (at least $P \geq 2$ ) compared to the LPA method using random excitation which might be impractical for some applications. Thus, the user needs to compromise between the importance of sorting out of the nonlinear distortion or conducting the experiment for a shorter time.

For the real plant, DRGA and its uncertainty bounds caused by the noise and the nonlinear distortions are estimated after the FRM of the $G_{B L A}$, the sample noise covariance $\left(C_{V}\right)$ and the covariance of the nonlinear distortions $\left(C_{Y_{s}}\right)$ have been estimated using LPA-FM method with dof equal to 7 . The results depicted Figure $9 \mathrm{~b}$ suggest similar pairing decision to result obtained using random excitation in Figure 9a. However, the values of $\hat{\lambda}_{11}$ and its uncertainty bounds are not identical, since $G_{B L A}$ depends on the amplitude distribution and the power spectrum of the excitation signal (Pintelon and Schoukens, 2012). Again, around a closed-loop bandwidth of $0.0012 \mathrm{~Hz}$ the system is expected to show good performance under a diagonal decentralized controller.

\section{Conclusion and Future Work}

In this paper a procedure to estimate the DRGA and its uncertainty bounds for weakly nonlinear systems using local polynomial approach is presented. The procedure allows the user to decide on the controller configuration without the need to rely on a parametric model. Local Polynomial Approximation (LPA) method was found to enhance DRGA estimation results compared to the Spectral Analysis (SA) method when the random excitations are imposed. Moreover, if the nonlinear distortions are to be estimated individually, Local Polynomial Approximation-Fast Method (LPA-FM) was found to shorten the experiment time using multisine excitation. Furthermore, in contrast to nonlinear RGA and DRGA based on the linearized model, estimated DRGA does not require an accurate system model for pairing decisions. Moreover, the estimation approach delivers the uncertainty bounds caused by the nonlinearities distortions and the measurement noise. The uncertainty bounds are useful to predict the magnitude of the interactions in specific frequencies. As for the practicality, even though RGA $(\Lambda(0))$ can be estimated easily using step response, it does not take the system dynamics into considerations and might lead to incorrect suggestions for the pairing decision as was shown in the case studies. Moreover, the step tests have to be applied sequentially for the MIMO systems which renders high costs in time and

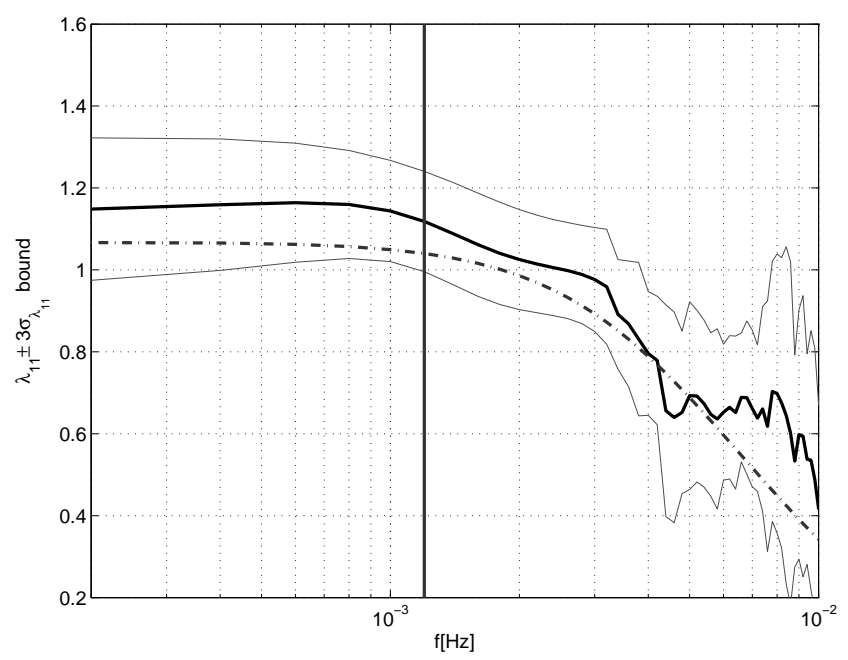

(a) Real parts of $\hat{\lambda}_{11}$ and $\pm 3 \sigma_{\lambda_{11}}$ bounds using LPA of the real case with random excitation. Black: $\hat{\lambda}_{11}$. light Gray: $\hat{\lambda}_{11} \pm 3 \sigma_{\lambda_{11}}$ uncertainty bounds. dark Gray (-.): $\lambda_{11}$ calculated based on $G_{l i n}$. Bold vertical line: indicates $0.0012 \mathrm{~Hz}$.

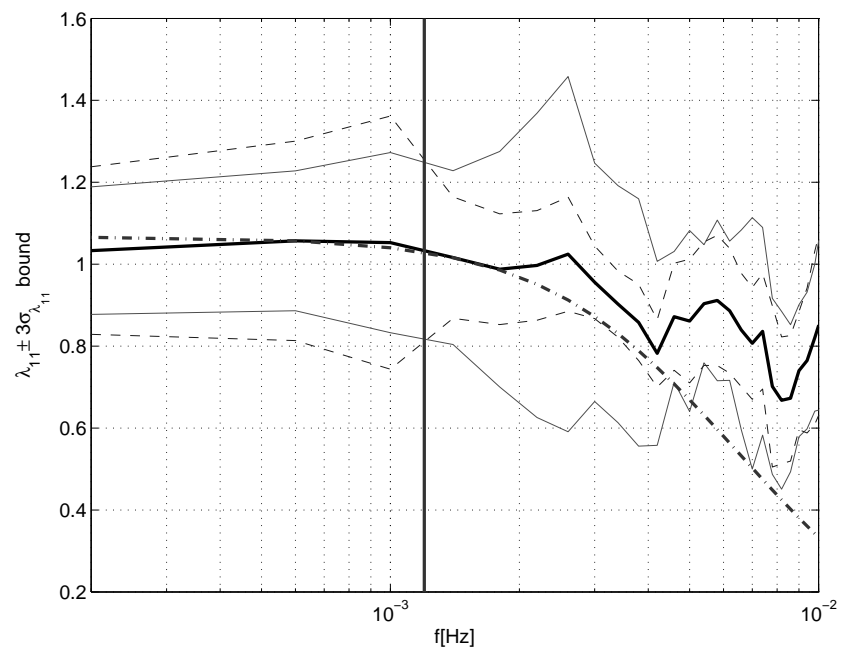

(b) Real parts of $\hat{\lambda}_{11}$ and $\pm 3 \sigma_{\lambda_{11}}$ bounds using LPM-FM of the real case with multisine excitation. Black: $\hat{\lambda}_{11}$. Gray (- -): $\hat{\lambda}_{11} \pm 3 \sigma_{\lambda_{11}}$ uncertainty bounds caused by noise. light Gray: $\hat{\lambda}_{11} \pm 3 \sigma_{\lambda_{11}}$ uncertainty bounds caused by nonlinear distortions. dark Gray (-·): $\lambda_{11}$ calculated based on $G_{l i n}$. Bold vertical line: indicates $0.0012 \mathrm{~Hz}$.

Figure 9: Real life results using LPA and LPA-FM methods. 
manpower whereas the excitation signals are applied simultaneously in the proposed procedure. Indeed, the nonparametric approach has its own difficulties to get informative results, still it is a very useful option to start with.

Towards more robust pairing decision, DRGA and its uncertainty bounds obtained by the proposed method can be utilized in future work to develop an algorithm for automatic configuration selection.

\section{References}

Antoni, J. and Schoukens, J. A comprehensive study of the bias and variance of frequency-response-function measurements: optimal window selection and overlapping strategies. Automatica, 2007. 43(10):17231736. doi:10.1016/j.automatica.2007.02.020.

Arranz, M. C. and Birk, W. Estimation of gramian-based interaction measures for weakly nonlinear systems. In ECC. pages 2438-2443, 2015. doi:10.1109/ECC.2015.7330904.

Bristol, E. On a new measure of interaction for multivariable process control. IEEE transactions on automatic control, 1966. pages 133-134.

Chen, D. and Seborg, D. E. Relative gain array analysis for uncertain process models. AIChE journal, 2002. 48(2):302-310. doi:10.1002/aic.690480214.

Dobrowiecki, T. P. and Schoukens, J. Linear approximation of weakly nonlinear mimo systems. IEEE Transactions on Instrumentation and Measurement, 2007. 56(3):887-894. doi:10.1109/TIM.2007.894898.

Glad, S. Extensions of the rga concept to nonlinear systems. 2000.

Goodwin, G. C., Graebe, S. F., and Salgado, M. E. Control system design, volume 240. Prentice Hall New Jersey, 2001.

Grosdidier, P., Morari, M., and Holt, B. R. Closedloop properties from steady-state gain information. Industrial \& engineering chemistry fundamentals, 1985. 24(2):221-235. doi:10.1021/i100018a015.

Johansson, K. H. The quadruple-tank process: A multivariable laboratory process with an adjustable zero. Control Systems Technology, IEEE Transactions on, 2000. 8(3):456-465. doi:10.1109/87.845876.

Kadhim, A. M., Birk, W., and Gustafsson, T. Relative gain array estimation based on non-parametric frequency domain system identification. In $C C A$. pages 110-115, 2014. doi:10.1109/CCA.2014.6981337.
Kadhim, A. M., Birk, W., and Gustafsson, T. Relative gain array variation for norm bounded uncertain systems. In CDC. pages 5959-5965, 2015a. doi:10.1109/CDC.2015.7403156.

Kadhim, A. M., Castano, M., Birk, W., and Gustafsson, T. Relative gain array of weakly nonlinear systems using nonparametric identification approach. In $C C A$. 2015b. doi:10.1109/CCA.2015.7320840.

Kariwala, V., Skogestad, S., and Forbes, J. F. Relative gain array for norm-bounded uncertain systems. Industrial \& engineering chemistry research, 2006. 45(5):1751-1757. doi:10.1021/ie050790r.

Khaki-Sedigh, A. and Moaveni, B. Control configuration selection for multivariable plants, volume 391. 2009. doi:10.1007/978-3-642-03193-9.

Moaveni, B. and Khaki-Sedigh, A. Input-output pairing for nonlinear multivariable systems. Journal of applied sciences, 2007. 7(22):3492-3498.

Pintelon, R. and Schoukens, J. System identification: a frequency domain approach. Wiley \& Sons, 2012.

Pintelon, R., Schoukens, J., Vandersteen, G., and Barbé, K. Estimation of nonparametric noise and frf models for multivariable systems-part i: Theory. Mech. Sys. \&6 Signal Proc., 2010. 24(3):573-595. doi:10.1016/j.ymssp.2009.08.009.

Schoukens, J., Marconato, A., Pintelon, R., Rolain, Y., Schoukens, M., Tiels, K., Vanbeylen, L., Vandersteen, G., and Van Mulders, A. System identification in a real world. In IEEE $A M C$ 2014. pages 1-9, 2014. doi:10.1109/AMC.2014.6823250.

Schoukens, J., Pintelon, R., and Rolain, Y. Mastering system identification in 100 exercises. 2012. doi:10.1002/9781118218532.

Schoukens, J., Pintelon, R., Vandersteen, G., and Rolain, Y. Design of excitation signals for nonparametric measurements on mimo-systems in the presence of nonlinear distortions. In IEEE IRMTC. pages 1-5, 2010. doi:10.1109/IMTC.2010.5488297.

Söderström, T. and Stoica, P. System identification. Prentice-Hall, Inc., 1988.

Wernholt, E. and Gunnarsson, S. Detection and estimation of nonlinear distortions in industrial robots. In Instr. \& Meas. Tech. Conf. pages 1913-1918, 2006. doi:10.1109/IMTC.2006.328310.

Witcher, M. and McAvoy, T. Interacting controlsystems-steady-state and dynamic measurement of interaction. ISA transactions, 1977. 16(3):35-41. 